\title{
Epilepsy surgery in children under 3 years of age: surgical and developmental outcomes
}

\author{
Masaki Iwasaki, MD, PhD, ${ }^{1}$ Keiya lijima, MD, PhD, ${ }^{1}$ Takahiro Kawashima, MEng, ${ }^{2}$ \\ Hisateru Tachimori, PhD, ${ }^{2}$ Yutaro Takayama, MD, ${ }^{1}$ Yuiko Kimura, MD, PhD, ${ }^{1}$ Yuu Kaneko, MD, ${ }^{1}$ \\ Naoki Ikegaya, MD, ${ }^{3}$ Noriko Sumitomo, MD, ${ }^{4}$ Takashi Saito, MD, ${ }^{4}$ Eiji Nakagawa, MD, PhD, ${ }^{4}$ \\ Akio Takahashi, MD, PhD, ${ }^{5}$ Kenji Sugai, MD, PhD, ${ }^{4}$ and Taisuke Otsuki, MD, PhD ${ }^{6}$

\begin{abstract}
'Department of Neurosurgery, National Center Hospital, National Center of Neurology and Psychiatry (NCNP), Kodaira, Tokyo; 2Department of Clinical Epidemiology, Translational Medical Center, NCNP, Kodaira, Tokyo; ${ }^{3}$ Department of Neurosurgery, Yokohama City University Hospital, Yokohama, Kanagawa; ${ }^{4}$ Department of Child Neurology, National Center Hospital, NCNP, Kodaira, Tokyo; ${ }^{5}$ Department of Neurosurgery, Shibukawa Medical Center, Shibukawa, Gunma; and ${ }^{6}$ Epilepsy Centre Bethel Japan, Iwanuma, Miyagi, Japan
\end{abstract}

\begin{abstract}
OBJECTIVE Pediatric epilepsy surgery is known to be effective, but early surgery in infancy is not well characterized. Extensive cortical dysplasia, such as hemimegalencephaly, can cause refractory epilepsy shortly after birth, and early surgical intervention is indicated. However, the complication rate of early pediatric surgery is significant. In this study, the authors assessed the risk-benefit balance of early pediatric epilepsy surgery as relates to developmental outcomes.

METHODS This is a retrospective descriptive study of 75 patients who underwent their first curative epilepsy surgery at an age under 3 years at the authors' institution between 2006 and 2019 and had a minimum 1-year follow-up of seizure and developmental outcomes. Clinical information including surgical complications, seizure outcomes, and developmental quotient $(\mathrm{DQ})$ was collected from medical records. The effects of clinical factors on $\mathrm{DQ}$ at 1 year after surgery were evaluated.
\end{abstract}

RESULTS The median age at surgery was 6 months, peaking at between 3 and 4 months. Operative procedures included 27 cases of hemispherotomy, 19 cases of multilobar surgery, and 29 cases of unilobar surgery. Seizure freedom was achieved in $82.7 \%$ of patients at 1 year and in $71.0 \%$ of patients at a mean follow-up of 62.8 months. The number of antiseizure medications (ASMs) decreased significantly after surgery, and 19 patients (30.6\%) had discontinued their ASMs by the last follow-up. Postoperative complications requiring cerebrospinal fluid (CSF) diversion surgery, such as hydrocephalus and cyst formation, were observed in 13 patients (17.3\%). The mean DQ values were $74.2 \pm 34.3$ preoperatively, $60.3 \pm 23.3$ at 1 year after surgery, and $53.4 \pm 25.1$ at the last follow-up. Multiple regression analysis revealed that the 1-year postoperative $D Q$ was significantly influenced by preoperative $D Q$ and postoperative seizure freedom but not by the occurrence of any surgical complication requiring CSF diversion surgery.

CONCLUSIONS Early pediatric epilepsy surgery has an acceptable risk-benefit balance. Seizure control after surgery is important for postoperative development.

https://thejns.org/doi/abs/10.3171/2021.4.PEDS21123

KEYWORDS epileptic encephalopathy; hemispherotomy; hemimegalencephaly; malformation of cortical development; developmental quotient; epilepsy

$\mathrm{S}$ URGICAL treatment for patients with drug-resistant focal epilepsy is efficacious in both adults and children. ${ }^{1,2}$ The primary goal of epilepsy surgery is to control a patient's seizures. Another important goal in pediatric patients is to prevent the cognitive and developmental stagnation or regression induced by persistent epilep- tic activity. Surgically treatable etiologies, such as hemimegalencephaly and focal cortical dysplasia, can cause severe seizures in infants, complicated by developmental epileptic encephalopathy such as early infantile epileptic encephalopathy or West syndrome. ${ }^{3}$

Infants with a large or hemispheric epileptogenic lesion

ABBREVIATIONS ASM = antiseizure medication; CSF = cerebrospinal fluid; $D Q=$ developmental quotient; $E E G=$ electroencephalography; ILAE = International League Against Epilepsy; KIDS = Kinder Infant Development Scale; PIH = periinsular hemispherotomy; Suzuki-Binet = Suzuki-Binet Intelligence Scale; Tanaka-Binet V = TanakaBinet Intelligence Scale, Fifth Edition; VPH = vertical parasagittal hemispherotomy; WISC-IV = Wechsler Intelligence Scale for Children, Fourth Edition; WPPSI = Wechsler Preschool and Primary Scale of Intelligence.

SUBMITTED March 2, 2021. ACCEPTED April 13, 2021.

INCLUDE WHEN CITING Published online August 13, 2021; DOI: 10.3171/2021.4.PEDS21123. 
may start to have seizures in the early neonatal period and require surgical intervention before 1 year of age. However, infantile epilepsy surgery has only been described in case series with a small or moderate number of subjects. A systematic literature review has suggested that the average chance of seizure freedom is $64 \%$, but the risk of perioperative complication is relatively high. In particular, the risk of hydrocephalus is as high as $20 \%$. Studies on developmental outcome after pediatric epilepsy surgery are further limited, although epilepsy surgery is believed to help patient development by controlling seizures. A shorter duration of epilepsy is known to be associated with greater developmental and cognitive gains after surgery., However, epilepsy surgery in infancy and early childhood is associated with significant surgical risks, so the benefit of early surgical intervention should be carefully investigated. We hypothesized that the benefits of early pediatric epilepsy surgery outweigh the risks in terms of a patient's later development.

In this study, we investigated whether a surgical complication, in particular hydrocephalus, had a negative impact on patient development. We summarize our institutional experience with epilepsy surgery in children under the age of 3 years and investigated the factors affecting developmental outcome.

\section{Methods}

This is a retrospective descriptive study. This paper was prepared in accordance with the STROBE guidelines. ${ }^{6}$ The study was approved by the ethics committee at the National Center of Neurology and Psychiatry, Tokyo, Japan.

\section{Patients}

This study included 75 patients who had undergone their first curative epilepsy surgery before the age of 3 years between August 2006 and February 2019 at our institution. Patients who had undergone palliative procedures, such as corpus callosotomy and vagus nerve stimulator implantation, were not included. The inclusion criteria were a minimum 1-year postoperative follow-up and a postoperative developmental assessment available either at 1 year or at the last follow-up after 2 years or longer. A total of 412 curative epilepsy surgeries were performed during the abovementioned period. Among them were 81 procedures that had been performed as the first surgery in patients younger than 3 years of age. Four patients with less than 1 year of follow-up were excluded. Two additional patients were excluded because no postoperative developmental assessments were available.

\section{Presurgical Evaluation}

Comprehensive presurgical evaluation including highfield MRI and long-term video-electroencephalography (EEG) monitoring was performed in all patients before surgery. Additional neuroimaging studies included interictal FDG-PET, ictal SPECT with technetium-99m ethyl cysteinate dimer, and magnetoencephalography. Surgical indication was determined in the patient management conference attended by pediatric neurologists, neurosur- geons, and certified epileptologists. Surgical treatment was indicated if drug-resistant seizures were present or highly expected based on the etiology of epilepsy.

\section{Surgery}

Surgery was generally considered for patients weighing more than $5 \mathrm{~kg}$ or older than 3 months. Perioperative management was performed by a team of pediatric neurologists and neurosurgeons. A central venous catheter was inserted preoperatively to secure routing for blood and/or fluid transfusion, if required.

Periinsular hemispherotomy (PIH) or vertical parasagittal hemispherotomy (VPH) was performed for hemispheric epileptogenic lesions such as hemimegalencephaly. The technical details of PIH were based on the Schramm and Villemure methods. ${ }^{7,8} \mathrm{VPH}$ was later introduced according to the method of Delalande et al.9,10 A technical modification was made as reported elsewhere and was applied in some patients. ${ }^{11}$ Posterior quadrantectomy was performed in patients with a posterior quadrantic epileptogenic lesion. The technical details are as previously reported. ${ }^{10,12}$ Lobectomy, lesionectomy, or focal cortical resection was performed for a focal epileptogenic lesion.

\section{Postoperative Complications}

Any acute or chronic surgery-related complication that unexpectedly required pharmacological or surgical interventions was identified. Any expected neurological deficits, such as hemiparesis after hemispherotomy, were not classified as complications. Blood transfusion was usually anticipated in surgery for patients under 1 year of age and so was not counted as a complication in this study. ${ }^{13}$

\section{Seizure Outcome}

Postoperative follow-up of the patients was achieved through outpatient visits or admissions for evaluations. Postoperative seizure outcome was assessed using the International League Against Epilepsy (ILAE) classification. $^{14}$

\section{Developmental Outcome}

Developmental status was assessed during preoperative evaluation and at postoperative outpatient visits or admissions. The postoperative assessment was performed at 3 months, 6 months, and 1 year after surgery and yearly thereafter. However, timing of the assessment after 1 year was highly variable depending on each patient's visits.

The Kinder Infant Development Scale (KIDS) was generally used for developmental assessment (Center of Developmental Education and Research, Tokyo, Japan, 1989). ${ }^{15-17}$ The KIDS consists of approximately 130 questions in Japanese answered by the parent and evaluating the child's development in 9 domains including motor function, manipulative function, receptive/expressive language functions, conceptualization, social relationships with children/adults, self-discipline, and feeding. The general developmental quotient (DQ) was calculated as the subject's summated developmental age across all domains divided by the chronological age. Enjoji's Development 
Scale ${ }^{18}$ and the Kyoto Scale of Psychological Development $2001^{15}$ were also used for evaluating infant's and children's DQ. DQ was replaced by IQ in the later follow-up of children, ${ }^{19,20}$ assessed using the Wechsler Intelligence Scale for Children, Fourth Edition (WISC-IV); Tanaka-Binet Intelligence Scale, Fifth Edition (Tanaka-Binet V); Suzuki-Binet Intelligence Scale (Suzuki-Binet); or Wechsler Preschool and Primary Scale of Intelligence (WPPSI).

\section{Data Collection}

Candidate patients were first identified from the National Center of Neurology and Psychiatry neurosurgical database. The following data were retrospectively collected from medical records: date of surgery; side and type of surgery; body weight at surgery; use of acute postoperative cerebrospinal fluid (CSF) drainage; epilepsy diagnosis; etiology of epilepsy; date of epilepsy onset; current or past epilepsy syndromes; previous history of surgical and medical treatment; seizure frequency; acute and chronic postoperative complications and dates of occurrence; date of the last follow-up; DQ before and 1 year after surgery and at the last follow-up, including the date and method of assessment; name and daily doses of antiseizure medications (ASMs) at the time of surgery, at 1 year after surgery, and at the last follow-up; date of terminating ASMs if applicable; postoperative seizure outcome at 1 year after surgery and at the last follow-up; date of postoperative seizure recurrence if applicable; and the date of, type of, and reason for reoperation if applicable. Postoperative seizures occurring within 1 week of surgery were not considered recurrences.

Assessments performed within 2 months of the 1st postoperative year were taken as the 1-year postoperative data. Assessments performed at least 2 years after surgery were used as the last follow-up data. Therefore, some patients had only 1-year data but no last follow-up data.

Types of surgery were classified into hemispheric, multilobar, and unilobar surgeries. Etiology of epilepsy was classified as hemimegalencephaly, tuberous sclerosis complex, other malformations of cortical development, tumor, Sturge-Weber syndrome, and ischemia.

\section{Statistical Analysis}

\section{Descriptive Statistics}

Descriptive statistics summarize the patient characteristics and postoperative course. The Kruskal-Wallis test and Mann-Whitney U-test with Bonferroni correction were used to compare the body weight of patients among surgical types. The Friedman test and Wilcoxon signedrank test with Bonferroni correction were used to examine the difference in the number of ASMs among the time of surgery, 1 year after surgery, and at the last follow-up. Kaplan-Meier survival analysis was used to estimate the probability of complete postoperative seizure freedom at 1,2 , and 5 years.

\section{Clinical Factors for DQ at 1 Year After Surgery}

Causal directed acyclic graphs were drawn, and clinical factors for multivariate analysis were chosen based on the backdoor criterion (Fig. S1). ${ }^{21,22}$ We considered the causal effects on postoperative development with a special interest in the effects of surgical complications requiring CSF diversion surgery, including the following 5 factors: preoperative DQ, seizure freedom at 1 year after surgery, type of surgery, type of etiology, and surgical complications requiring CSF diversion surgery. Pre- and postoperative DQ included missing values because of a lack of assessments within specific periods, as mentioned above. Missing values in DQ were interpolated using the multivariate imputation by chained equations (MICE) with 1000 multiply imputed data sets. Complete case analysis was also executed with a similar configuration for comparison. Multiple linear regression analysis was used to assess the relationship between the above 5 variables and DQ at 1 year after surgery. $R$ version 4.0.0 and above (The $\mathrm{R}$ Foundation for Statistical Computing) and R MICE package version 3.10.0 were used for the statistical analysis. Statistical significance was accepted at $\mathrm{p}<0.05$.

\section{Results \\ Patient Characteristics}

Of the 75 patients included in our analysis, 39 were female and 36 were male, with an age range of 58 days to 35 months at surgery (mean $11.9 \pm 10.8$ months, median 6 months). Age at surgery was not normally distributed, peaking at between 3 and 4 months (Fig. 1). Body weight at surgery was $4.08-15.8 \mathrm{~kg}$ (mean $8.88 \pm 3.03 \mathrm{~kg}$ ). Age at epilepsy onset was $0-830$ days (mean $110 \pm 188$ days). Duration of epilepsy was 1-35 months (mean $8.3 \pm 7.9$ months). Number of ASMs at the time of surgery was 1-6 (mean 2.21). The etiology of epilepsy was hemimegalencephaly in 22 patients, other malformations of cortical development in 33, low-grade developmental tumors in 10, tuberous sclerosis complex in 6, Sturge-Weber syndrome in 3 , and perinatal ischemia in 1 . Ohtahara syndrome and West syndrome were diagnosed in 8 and 15 patients, respectively. Sixty-eight patients had daily seizures, and 6 patients had weekly seizures. One patient with Sturge-Weber syndrome presented with rare clinical seizures before surgery.

\section{Surgery}

Hemispherotomy, multilobar surgery, and unilobar surgery were performed in $27(36 \%), 19(25 \%)$, and $29(39 \%)$ patients, respectively (Table 1). Delalande's vertical hemispherotomy was the dominant procedure for hemispheric disconnection surgery. No patient underwent anatomical hemispherectomy. Chronic intracranial EEG monitoring with subdural electrodes was performed before lesionectomy in a 32-month-old patient with tuberous sclerosis complex.

Body weight was significantly different among the types of surgery ( $p<0.001$, Kruskal-Wallis test). Body weight was lower in the patients who had undergone hemispherotomy $(6.99 \pm 2.00 \mathrm{~kg})$ and multilobar surgery $(8.32$ $\pm 2.07 \mathrm{~kg}$ ) than in those who had undergone unilobar surgery $(11.0 \pm 3.07 \mathrm{~kg} ; \mathrm{p}<0.001$ and $\mathrm{p}=0.007$, respectively, Mann-Whitney U-test with Bonferroni correction). A CSF drainage tube was placed in the lateral ventricle or surgical cavity at the end of surgery and external CSF drainage 


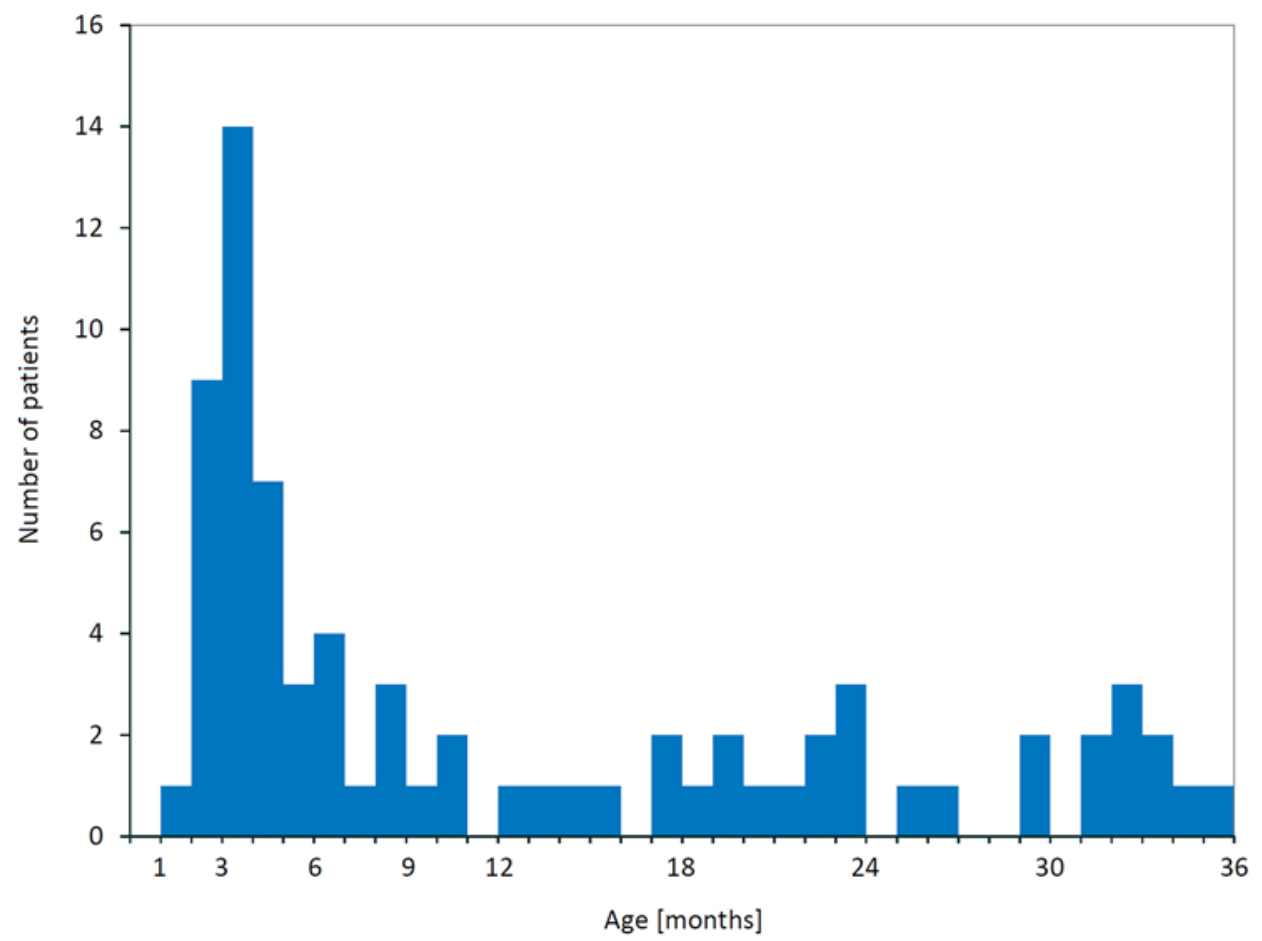

FIG. 1. Histogram showing the distribution of patients by age at surgery. The most common age at surgery was between 3 and 4 months. Figure is available in color online only.

was performed for 1 or 2 postoperative days in 29 patients (38.7\%), including 18 after hemispherotomy, 9 after multilobar surgery, and 2 after frontal lobectomy. ${ }^{23}$

Repeat epilepsy surgery was performed in 12 patients (16.0\%) during the follow-up period, including in 2 patients who underwent 3 surgeries. Additional cortical resection was performed once in 4 patients and twice in 1 patient.
Posterior quadrantic disconnection was performed in 1 patient at 57 months after the first focal cortical resection. Hemispherotomy was performed in 1 patient at 13 months after the first surgery, following 2 multilobar cortical resection procedures. Disconnection of the splenium was performed for incomplete hemispherotomy or posterior quadrantic disconnection in 3 patients. Two patients un-

TABLE 1. Postoperative CSF drainage and surgical complications by type of surgery

\begin{tabular}{|c|c|c|c|c|c|c|}
\hline \multirow[b]{2}{*}{ Type of Surgery } & \multirow{2}{*}{$\begin{array}{l}\text { No. of } \\
\text { Cases }\end{array}$} & \multirow{2}{*}{$\begin{array}{c}\text { CSF } \\
\text { Drainage }\end{array}$} & \multicolumn{2}{|r|}{$\begin{array}{c}\text { Complications Requiring } \\
\text { Medical Treatment }\end{array}$} & \multicolumn{2}{|c|}{$\begin{array}{l}\text { Complications Requiring } \\
\text { Surgical Intervention }\end{array}$} \\
\hline & & & Total No. & Detail (no. of cases) & Total No. & Detail (no. of cases) \\
\hline \multicolumn{7}{|l|}{ Hemispherotomy $(n=27)$} \\
\hline VPH & 22 & 14 & 4 & $\begin{array}{l}\text { CSWS (2), DI (3), sinus thrombosis (2), } \\
\text { asymptomatic hemorrhagic infarction (1) }\end{array}$ & 7 & $\begin{array}{l}\text { Cyst formation (2), } \\
\text { hydrocephalus (5) }\end{array}$ \\
\hline $\mathrm{PIH}$ & 5 & 4 & 0 & & 1 & Hydrocephalus (1) \\
\hline \multicolumn{7}{|l|}{ Multilobar surgery $(n=19)$} \\
\hline Subtotal hemispherotomy & 1 & 0 & 0 & & 0 & \\
\hline Posterior quadrantic disconnection & 13 & 8 & 1 & Asymptomatic cerebral infarction (1) & 1 & Cyst formation (1) \\
\hline Multilobar cortical resection & 5 & 1 & 0 & & 2 & $\begin{array}{l}\text { Subdural hygroma (1), } \\
\text { cyst formation (1) }\end{array}$ \\
\hline \multicolumn{7}{|l|}{ Unilobar surgery $(n=29)$} \\
\hline Anterior temporal lobectomy & 8 & 0 & 1 & Psychiatric symptom (1) & 0 & \\
\hline Frontal lobectomy or disconnection & 5 & 2 & 1 & Bacterial meningitis (1) & 1 & Subdural hygroma (1) \\
\hline $\begin{array}{l}\text { Focal cortical resection or lesion- } \\
\text { ectomy }\end{array}$ & 16 & 0 & 0 & & 1 & Subdural hygroma (1) \\
\hline
\end{tabular}

CSWS = cerebral salt wasting syndrome; $\mathrm{DI}=$ diabetes insipidus. 
TABLE 2. Postoperative seizure outcome

\begin{tabular}{ccc}
\hline & \multicolumn{2}{c}{ No. of Patients $(\%)$} \\
\cline { 2 - 3 } ILAE Class & 1 Year $(n=75)$ & Last FU $(n=62)$ \\
\hline 1 & $62(82.7)$ & $44(71.0)$ \\
\hline 2 & $1(1.3)$ & 0 \\
\hline 3 & $2(2.7)$ & $6(9.7)$ \\
\hline 4 & $6(8.0)$ & $9(14.5)$ \\
\hline 5 & $4(5.3)$ & $2(3.2)$ \\
\hline 6 & 0 & $1(1.6)$ \\
\hline
\end{tabular}

$\mathrm{FU}=$ follow-up.

derwent corpus callosotomy as a palliative procedure 10 and 25 months after the first surgery. Seven surgeries in 6 patients were performed within 1 year of the first surgery.

\section{Seizure Outcome}

Postoperative seizure outcome is shown in Table 2. The rate of seizure freedom was $82.7 \%$ at 1 year after surgery and $71.0 \%$ at the last follow-up after 2 or more years. The mean follow-up period was $62.8 \pm 30.4$ months $(24-155$ months). Figure 2 shows the Kaplan-Meier curve for postoperative seizure outcome. The rate of complete postoper- ative seizure freedom was $78.7 \%$ (95\% CI 0.676-0.863) at 1 year, $69.8 \%$ (95\% CI $0.577-0.790)$ at 2 years, and $58.4 \%$ (95\% CI 0.451-0.695) at 5 years. Of the 62 patients with 2 or more years' follow-up, 27 experienced postoperative seizures. However, 9 of the 27 patients attained a class 1 outcome by the time of the last follow-up with additional treatments, such as medication adjustment and repeat surgery.

The number of ASMs was significantly decreased after surgery. Patients were taking an average 2.21 ASMs (range $1-6, \mathrm{n}=75$ ) at the time of surgery, 1.85 ASMs (range $0-4, \mathrm{n}=74$ ) at 1 year after surgery, and 1.32 ASMs (range $0-5, \mathrm{n}=62$ ) at the last follow-up. The difference was significant between at surgery and 1 year after surgery $(\mathrm{p}=0.003$, Wilcoxon signed-rank test $)$ and between 1 year after surgery and at the last follow-up $(\mathrm{p}<0.001)$. Three and 19 patients (30.6\%) were not taking any ASMs at 1 year after surgery and at the last follow-up, respectively. Follow-up duration after ASM discontinuation was a mean 21.1 months (range 0-59 months).

\section{Surgical Complications}

No deaths were observed. Twenty surgical complications occurred in 19 patients, most frequently after hemispherotomy. The details are summarized in Table 1. Diabetes insipidus and cerebral salt wasting syndrome were

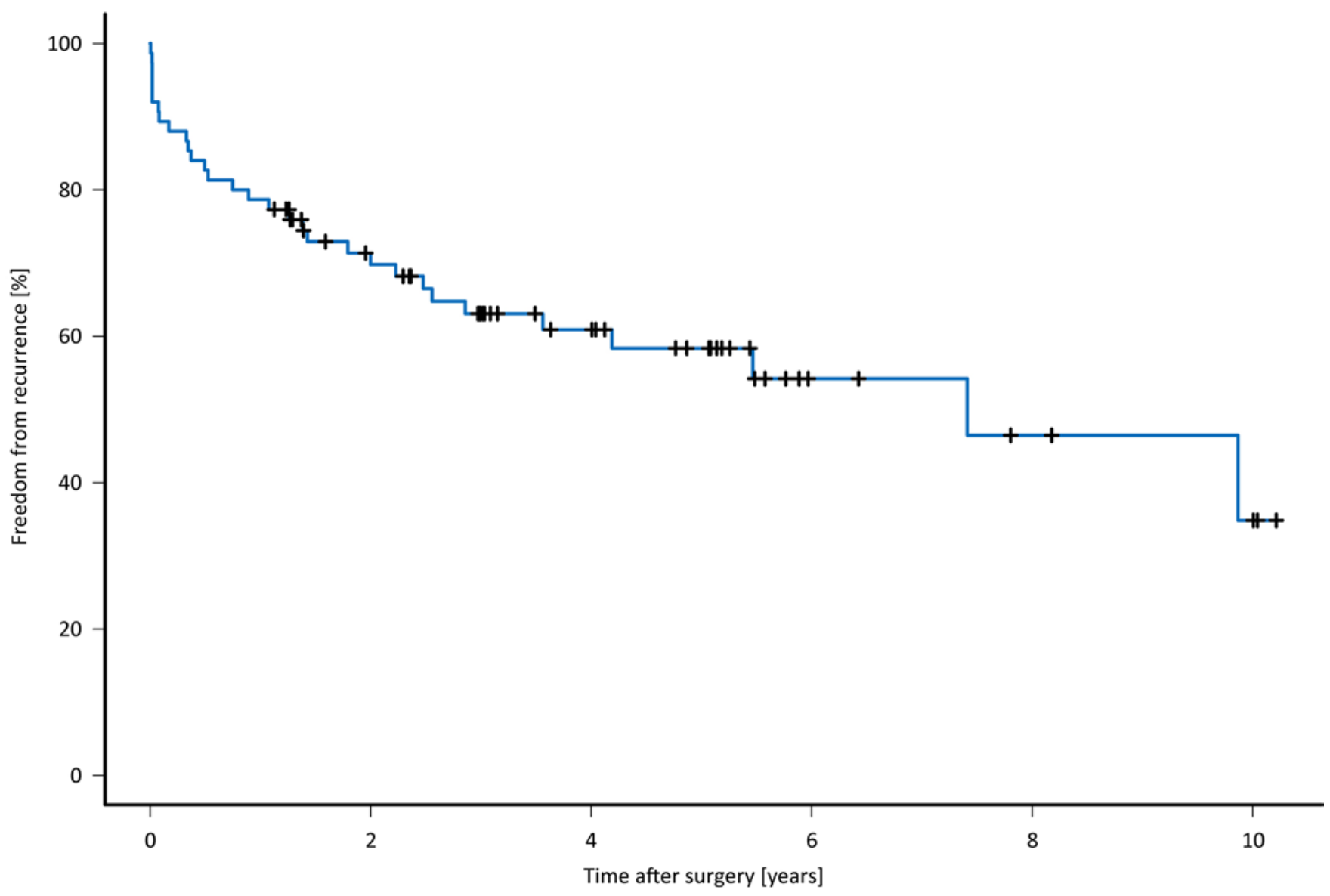

FIG. 2. Kaplan-Meier curve showing the rate of complete postoperative seizure freedom. Figure is available in color online only. 
TABLE 3. Multiple regression analysis for DQ 1 year after surgery

\begin{tabular}{lrrr}
\hline \multicolumn{1}{c}{ Variable } & Estimate & $\begin{array}{r}\text { Standard } \\
\text { Deviation }\end{array}$ & $\begin{array}{c}\mathrm{p} \\
\text { Value }\end{array}$ \\
\hline Intercept & 14.785 & 10.094 & 0.148 \\
\hline Preop DQ & 0.369 & 0.065 & $<0.001$ \\
\hline Postop seizure freedom & 19.532 & 6.249 & $\mathbf{0 . 0 0 3}$ \\
\hline Type of surgery: unilobar surgery & 7.014 & 5.489 & 0.206 \\
\hline Type of surgery: multilobar surgery & 2.894 & 5.763 & 0.617 \\
\hline Etiology: malformation of cortical & -2.659 & 6.164 & 0.668 \\
development & & & \\
\hline CSF diversion surgery & -0.269 & 6.151 & 0.965 \\
\hline
\end{tabular}

Boldface type indicates statistical significance.

observed only after hemispherotomy. Two cases of sinus thrombosis occurred secondary to diabetes insipidus, as reported separately. ${ }^{24}$ All complications that required medical treatment were transient, and no permanent sequela was observed.

Cystic enlargement of the postoperative cavity or hydrocephalus with or without subdural fluid collection occurred in 13 patients (17.3\%). All of these patients were treated with CSF diversion surgery, including ventriculoperitoneal shunt placement in 6 patients, cyst-peritoneal shunt insertion in 3, subdural-peritoneal shunt placement in 2 , and cyst fenestration in 2 . The symptom was often subacute, and the median interval between epilepsy surgery and CSF diversion was 4 months (range 26 days -30 months). Shunting surgery was preceded by endoscopic or open fenestration of the cyst wall, septum pellucidum, or third ventricle floor in 4 patients. Shunt revision surgery was performed during the follow-up period in 2 patients. Shunt infection occurred in 1 patient 3 weeks after surgery; hydrocephalus did not recur after shunt removal in this patient.

\section{Developmental Outcome}

The mean DQ values were $74.15 \pm 34.34$ before surgery $(n=68), 60.34 \pm 23.31$ at 1 year after surgery $(n=$ $68)$, and $53.43 \pm 25.06$ at the last follow-up $(n=58)$. Other unassessed outcomes were treated as missing values. DQ before surgery was assessed with the KIDS in 67 patients and Enjoji's Development Scale in 1 patient. DQ at 1 year after surgery was assessed with the KIDS in 64 patients and Enjoji's Scale in 4. The last follow-up DQ was assessed using the KIDS in 48 patients, Enjoji's Scale in 1, and the Kyoto Scale of Psychological Development 2001 in 1. DQ was substituted by IQ in the other 8 patients (WISC-IV in 4, Tanaka-Binet V in 2, Suzuki-Binet in 1, WPPSI in 1).

\section{Clinical Factors for Postoperative DQ}

Table 3 summarizes the results of multiple regression analysis. DQ at 1 year after surgery was significantly influenced by DQ before surgery and postoperative seizure freedom but not by any surgical complication requiring CSF diversion surgery. The results of our complete case analysis also indicated that surgical complication requiring CSF diversion was not significantly important (Table S1).

\section{Discussion}

This study showed that surgery in the early pediatric stage is effective for controlling epileptic seizures and reducing the requirement for ASMs. Complete seizure freedom was achieved in $82.7 \%$ of patients at 1 year after surgery and in $71.0 \%$ at the last follow-up 2 or more years after surgery. ASMs were no longer required in $30.6 \%$ of patients at the last follow-up. The risk of hydrocephalus or cystic enlargement of the surgical cavity requiring CSF diversion surgery was as high as $17.3 \%$. No deaths were observed. Postoperative development at 1 year after surgery was dependent on the preoperative DQ and postoperative seizure freedom but not on the occurrence of surgical complications requiring CSF diversion. These findings suggest that early epilepsy surgery in pediatric patients has an acceptable risk-benefit balance.

The present series contains a large number of cases of epilepsy surgery in children under 3 years of age. Hemimegalencephaly and extensive cortical dysplasia can cause severe neonatal-onset epileptic encephalopathy and indicate surgery in the 1st year of life. ${ }^{25,26}$ Earlier seizure control is better for early development, whereas early surgery is compromised by associated risks because the major procedures (i.e., hemispheric or multilobar surgeries) are common in this age group. Safe methods of early surgery are not well characterized. Our criteria for an early surgical indication are a body weight of $5 \mathrm{~kg}$ or more and/or an age of 3 months or older, but these are solely based on our experience. Certain caution must be exercised in the application of these criteria. Very careful manipulation is necessary in surgery in infants, especially those under the age of 6 months, because the pia-arachnoid membrane and venous vessel walls are relatively fragile and meticulous care is often required to achieve hemostasis. This study suggests that epilepsy surgery at age 3 or 4 months can be performed relatively safely by a team of experienced neurosurgeons and pediatric neurologists.

Seizure freedom after epilepsy surgery is associated with better postoperative development. However, postoperative development after epilepsy surgery in infancy and early childhood has not been thoroughly assessed. We previously reported that seizure freedom was associated with a better developmental outcome after hemispherotomy in a series of 12 consecutive patients presenting with Ohtahara syndrome or West syndrome. ${ }^{26}$ Good developmental progress as measured by the social interaction quotient was most evident in the infants who were seizure free and without medication among the 20 patients who had undergone epilepsy surgery before the age of 36 months. ${ }^{27}$

The biggest factor in determining postoperative development is preoperative development, ${ }^{4,28}$ which is largely determined by the size of the epileptogenic lesion and the duration of epilepsy. A shorter duration of epilepsy is associated with better postoperative development. ${ }^{29-31}$ Epilepsy surgery before the age of 12 months has been correlated with improvement in DQ as assessed with the Bayley Scales of Infant Development. ${ }^{4}$ Recent investigation into adaptive and cognitive development in 48 cases after infantile epilepsy surgery revealed that a longer epilepsy duration and larger lesion determined postsurgical developmental outcome..$^{5}$ Average DQ after surgery was 60 or 
lower in the current study, suggesting that developmental growth after surgery is often limited and that many infants fail to catch up with "normal" progress. This finding was likely attributable to the fact that most patients had large epileptogenic lesions and underwent multilobar or hemispheric surgery. Later development depends on the amount of preserved functioning brain. Full development cannot be expected if only a few functional brain regions remain. However, even within this constraint, seizure control by surgical treatment is beneficial for patient development.

Better development after seizure freedom can be partly attributed to a reduction or discontinuation of ASMs. In this study, we found that ASMs were significantly reduced after surgery and were discontinued in $30.6 \%$ of patients. ASMs can be discontinued after epilepsy surgery in infancy and early childhood in $20 \%-40 \%$ of patients. . $^{5,32,33}$ A reduction in the number of ASMs and complete ASM withdrawal are independent determinants of cognitive gains after pediatric epilepsy surgery. ${ }^{19}$ One multicenter observational cohort study has revealed that resective surgeries are associated with better seizure and developmental outcomes than those obtained with successful medical treatment in children with epileptic encephalopathy. ${ }^{34}$

The chance of seizure freedom after epilepsy surgery is higher in children than in adults. ${ }^{35-37} \mathrm{~A}$ systematic review and meta-analysis has revealed that a shorter epilepsy duration is associated with a better chance of seizure freedom after surgery in general. ${ }^{38}$ Therefore, the chance of seizure freedom may be higher in infants than in older children/adolescents. ${ }^{36}$ The present study demonstrated an ILAE class 1 outcome in $83 \%$ of patients at 1 year after surgery and in $71 \%$ at the last follow-up 2 or more years after surgery. Such outcomes are comparable to or even better than previous results of epilepsy surgery in children under 3 years of age. A systematic literature review demonstrated, on average, a $64 \%$ rate of seizure freedom in infantile epilepsy surgery, with a range from $46 \%$ to $89 \%{ }^{33,39-41}$ However, whether earlier surgery is associated with better seizure outcome in infants with epilepsy should be further clarified. The mean age at surgery was younger in our study than in most previous reports.

Epilepsy surgery in early childhood carries a relatively high risk of surgical complication. Death has been rarely reported. The risk of hydrocephalus has been reported as $5 \%$ to $20 \%$ in epilepsy surgery under 3 years of age..$^{5,40}$ The risk is higher in patients undergoing hemispherotomy/hemispherectomy, which requires extensive exposure of the lateral ventricle. One multiinstitutional survey has revealed that $23 \%$ of 690 patients required hydrocephalus treatment after hemispherectomy and that anatomical hemispherectomy was an independent risk factor for developing hydrocephalus. ${ }^{42}$ Our risk of hydrocephalus requiring CSF diversion surgery (17.3\%) was relatively high compared with previous reports, probably because our series included many young patients. A low body weight was associated with the risk of hydrocephalus in our study, and young infants frequently undergo hemispherotomy. Therefore, whether low body weight is specifically a risk for postoperative hydrocephalus should be carefully considered.

The major limitation of this study is its single-center retrospective design, which only provides a low level of evidence. The efficacy of pediatric epilepsy surgery for carefully selected patients has been established. However, surgery in very early life, such as before 6 months of age, may require certain expertise among neurosurgeons and pediatric neurologists. The risk-benefit assessment of this approach should be reproduced at multiple institutions. Ideally, a controlled study is necessary to claim the riskbenefit balance of our approach with a high level of evidence. Moreover, different procedures and etiologies are analyzed together in this study. The risk-benefit balance should be further elucidated for each etiology and type of procedure based on sufficient numbers of subjects. The trajectory of DQ with the time around surgery should have been investigated to understand whether epilepsy surgery arrested developmental decline. However, our study could not focus on this aspect because the accurate assessment of development was relatively difficult in young infants and because the trend of DQ was not available for most patients during the short period before surgery. The effect of surgical treatment on different aspects of development should be studied. For example, hemiparesis after hemispherotomy would impair motor milestones of patients, resulting in lower global development. Multiple regression analysis was limited to development at 1 year after surgery in this study because regular developmental followup after 2 or more years was not available. Cessation of epileptic activity after early surgery can be followed by substantial cognitive gains in the short term. However, longer follow-up revealed stabilization of cognitive levels in the impaired range in most children. ${ }^{43}$ Further study on the long-term outcome is necessary.

\section{Conclusions}

Epilepsy surgery in the early pediatric stage is effective for controlling epileptic seizures and reducing the requirement for ASMs. The risk of hydrocephalus or cystic enlargement of the surgical cavity requiring CSF diversion surgery is as high as $17.3 \%$. Postoperative development at 1 year after surgery was dependent on preoperative development and postoperative seizure freedom but not on the occurrence of surgical complications requiring CSF diversion surgery. Thus, early pediatric epilepsy surgery has an acceptable risk-benefit balance. Seizure control after surgery is important for postoperative development.

\section{Acknowledgments}

This study was supported, in part, by Grants-in-Aid for Scientific Research (KAKENHI) grant no. JP19K09494 from the Japan Society for the Promotion of Science (JSPS), by the Japan Agency for Medical Research and Development (AMED) under grant nos. JP20ek0109374 and JP20ck0106534, and by Intramural Research Grant (1-4: Integrative research on pathomechanism, diagnostic methodology and therapeutics for epilepsy) for Neurological and Psychiatric Disorders of the National Center of Neurology and Psychiatry.

\section{References}

1. Dwivedi R, Ramanujam B, Chandra PS, et al. Surgery for drug-resistant epilepsy in children. N Engl J Med. 2017; 377(17):1639-1647. 
2. Wiebe S, Blume WT, Girvin JP, Eliasziw M. A randomized, controlled trial of surgery for temporal-lobe epilepsy. $N$ Engl J Med. 2001;345(5):311-318.

3. Di Rocco C, Battaglia D, Pietrini D, et al. Hemimegalencephaly: clinical implications and surgical treatment. Childs Nerv Syst. 2006;22(8):852-866.

4. Loddenkemper T, Holland KD, Stanford LD, et al. Developmental outcome after epilepsy surgery in infancy. Pediatrics. 2007;119(5):930-935.

5. Kadish NE, Bast T, Reuner G, et al. Epilepsy surgery in the first 3 years of life: predictors of seizure freedom and cognitive development. Neurosurgery. 2019;84(6):E368-E377.

6. von Elm E, Altman DG, Egger M, et al. The Strengthening the Reporting of Observational Studies in Epidemiology (STROBE) statement: guidelines for reporting observational studies. Lancet. 2007;370(9596):1453-1457.

7. Villemure JG, Mascott CR. Peri-insular hemispherotomy: surgical principles and anatomy. Neurosurgery. 1995;37(5): 975-981.

8. Schramm J, Behrens E, Entzian W. Hemispherical deafferentation: an alternative to functional hemispherectomy. Neurosurgery. 1995;36(3):509-516.

9. Delalande O, Bulteau C, Dellatolas G, et al. Vertical parasagittal hemispherotomy: surgical procedures and clinical longterm outcomes in a population of 83 children. Neurosurgery. 2007;60(2)(suppl 1):ONS19-ONS32.

10. Otsuki T. Hemispherotomy and multilobar surgery. In: Arzimanoglou A, Cross JH, Gaillard WD, et al, eds. Pediatric Epilepsy Surgery. John Libbey Eurotext; 2016:387-397.

11. Iwasaki M, Uematsu M, Osawa SI, et al. Interhemispheric vertical hemispherotomy: a single center experience. Pediatr Neurosurg. 2015;50(5):295-300.

12. Daniel RT, Meagher-Villemure K, Farmer JP, et al. Posterior quadrantic epilepsy surgery: technical variants, surgical anatomy, and case series. Epilepsia. 2007;48(8):1429-1437.

13. Gowda S, Salazar F, Bingaman WE, et al. Surgery for catastrophic epilepsy in infants 6 months of age and younger. $J$ Neurosurg Pediatr. 2010;5(6):603-607.

14. Wieser HG, Blume WT, Fish D, et al. ILAE Commission Report. Proposal for a new classification of outcome with respect to epileptic seizures following epilepsy surgery. Epilepsia. 2001;42(2):282-286.

15. Aoki S, Hashimoto K, Ikeda N, et al. Comparison of the Kyoto Scale of Psychological Development 2001 with the parent-rated Kinder Infant Development Scale (KIDS). Brain Dev. 2016;38(5):481-490.

16. Cheng S, Maeda T, Tomiwa K, et al. Contribution of parenting factors to the developmental attainment of 9-month-old infants: results from the Japan Children's Study. J Epidemiol. 2009;19(6):319-327.

17. Baba H, Toda K, Ono T, et al. Surgical and developmental outcomes of corpus callosotomy for West syndrome in patients without MRI lesions. Epilepsia. 2018;59(12):22312239.

18. Enjoji M, Gouya N, Kurokawa T, et al. Enjoji Scale of Infant Analytical Development Manual. Keio University Press; 2009.

19. Boshuisen K, van Schooneveld MMJ, Uiterwaal CSPM, et al. Intelligence quotient improves after antiepileptic drug withdrawal following pediatric epilepsy surgery. Ann Neurol. 2015;78(1):104-114.

20. Ikegaya N, Iwasaki M, Kaneko Y, et al. Cognitive and developmental outcomes after pediatric insular epilepsy surgery for focal cortical dysplasia. J Neurosurg Pediatr. 2020;26(5): 543-551.

21. Pearl J. Causal diagrams for empirical research. Biometrika. 1995;82(4):669-688

22. Greenland S, Pearl J, Robins JM. Causal diagrams for epidemiologic research. Epidemiology. 1999;10(1):37-48.
23. Sood S, Asano E, Chugani HT. Role of external ventriculostomy in the management of fever after hemispherectomy. $J$ Neurosurg Pediatr. 2008;2(6):427-429.

24. Saito T, Sugai K, Takahashi A, et al. Transient water-electrolyte disturbance after hemispherotomy in young infants with epileptic encephalopathy. Childs Nerv Syst. 2020;36(5): 1043-1048.

25. Sasaki M, Hashimoto T, Furushima W, et al. Clinical aspects of hemimegalencephaly by means of a nationwide survey. $J$ Child Neurol. 2005;20(4):337-341.

26. Honda R, Kaido T, Sugai K, et al. Long-term developmental outcome after early hemispherotomy for hemimegalencephaly in infants with epileptic encephalopathy. Epilepsy Behav. 2013;29(1):30-35.

27. Gröppel G, Dorfer C, Dressler A, et al. Epilepsy surgery in infants: safety issues and developmental outcome. Wien Klin Wochenschr. 2018;130(9-10):341-348.

28. Ramantani G, Kadish NE, Strobl K, et al. Seizure and cognitive outcomes of epilepsy surgery in infancy and early childhood. Eur J Paediatr Neurol. 2013;17(5):498-506.

29. Jonas R, Asarnow RF, LoPresti C, et al. Surgery for symptomatic infant-onset epileptic encephalopathy with and without infantile spasms. Neurology. 2005;64(4):746-750.

30. Basheer SN, Connolly MB, Lautzenhiser A, et al. Hemispheric surgery in children with refractory epilepsy: seizure outcome, complications, and adaptive function. Epilepsia. 2007;48(1):133-140.

31. Freitag H, Tuxhorn I. Cognitive function in preschool children after epilepsy surgery: rationale for early intervention. Epilepsia. 2005;46(4):561-567.

32. Reinholdson J, Olsson I, Edelvik A, et al. Long-term followup after epilepsy surgery in infancy and early childhood-a prospective population based observational study. Seizure. 2015;30:83-89.

33. Kumar RM, Koh S, Knupp K, et al. Surgery for infants with catastrophic epilepsy: an analysis of complications and efficacy. Childs Nerv Syst. 2015;31(9):1479-1491.

34. Otsuki T, Kim HD, Luan G, et al. Surgical versus medical treatment for children with epileptic encephalopathy in infancy and early childhood: results of an international multicenter cohort study in Far-East Asia (the FACE study). Brain Dev. 2016;38(5):449-460.

35. d'Orio P, Rizzi M, Mariani V, et al. Surgery in patients with childhood-onset epilepsy: analysis of complications and predictive risk factors for a severely complicated course. $J$ Neurol Neurosurg Psychiatry. 2019;90(1):84-89.

36. Jenny B, Smoll N, El Hassani Y, et al. Pediatric epilepsy surgery: could age be a predictor of outcomes? J Neurosurg Pediatr. 2016;18(2):235-241.

37. Cloppenborg T, May TW, Blümcke I, et al. Differences in pediatric and adult epilepsy surgery: a comparison at one center from 1990 to 2014. Epilepsia. 2019;60(2):233-245.

38. Bjellvi J, Olsson I, Malmgren K, Wilbe Ramsay K. Epilepsy duration and seizure outcome in epilepsy surgery: a systematic review and meta-analysis. Neurology. 2019;93(2):e159e166.

39. Pindrik J, Hoang N, Smith L, et al. Preoperative evaluation and surgical management of infants and toddlers with drugresistant epilepsy. Neurosurg Focus. 2018;45(3):E3.

40. Dunkley C, Kung J, Scott RC, et al. Epilepsy surgery in children under 3 years. Epilepsy Res. 2011;93(2-3):96-106.

41. Steinbok P, Gan PYC, Connolly MB, et al. Epilepsy surgery in the first 3 years of life: a Canadian survey. Epilepsia. 2009;50(6):1442-1449.

42. Lew SM, Matthews AE, Hartman AL, Haranhalli N. Posthemispherectomy hydrocephalus: results of a comprehensive, multiinstitutional review. Epilepsia. 2013;54(2):383-389.

43. Roulet-Perez E, Davidoff V, Mayor-Dubois C, et al. Impact of severe epilepsy on development: recovery potential after 
successful early epilepsy surgery. Epilepsia. 2010;51(7): $1266-1276$

\section{Disclosures}

Dr. Iwasaki has received speaker honoraria from Daiichi Sankyo Co. Ltd. and contract research expenses from UCB Japan Co. Ltd., Eisai Co. Ltd., and LivaNova Japan K.K. Dr. Nakagawa has received speaker honoraria from Nobelpharma Co. Ltd. Dr. Sugai has received speaker honoraria from Eisai Co. Ltd.

\section{Author Contributions}

Conception and design: Iwasaki. Acquisition of data: Iwasaki, Iijima, Takayama, Kimura, Ikegaya, Sumitomo, Saito, Takahashi. Analysis and interpretation of data: Iwasaki, Iijima, Kawashima. Drafting the article: Iwasaki. Critically revising the article: Iwasaki, Kawashima, Tachimori, Sumitomo, Sugai, Otsuki. Reviewed submitted version of manuscript: Iijima, Tachimori, Takayama, Kimura, Kaneko, Ikegaya, Sumitomo, Saito,
Nakagawa, Takahashi, Sugai. Approved the final version of the manuscript on behalf of all authors: Iwasaki. Statistical analysis: Iwasaki, Iijima, Kawashima, Tachimori. Study supervision: Nakagawa, Sugai, Otsuki.

\section{Supplemental Information}

Online-Only Content

Supplemental material is available with the online version of the article.

Figure S1 and Table S1. https://thejns.org/doi/suppl/10.3171/ 2021.4.PEDS21123.

\section{Correspondence}

Masaki Iwasaki: National Center Hospital, National Center of Neurology and Psychiatry, Tokyo, Japan. iwa@ncnp.go.jp. 